\title{
Quasi-total backward reflection with a CRIGF under oblique incidence
}

\author{
Francois Renaud Gamal Mohamed \\ Anne-Laure Fehrembach Evgueni Popov \\ Antoine Monmayrant Olivier Gauthier-Lafaye
}

Cite this manuscript as : Renaud, F., Mohamed, G., Fehrembach, AL. et al. Quasi-total backward reflection with a CRIGF under oblique incidence. Opt Quant Electron 52, 184 (2020). https://doi.org/10.1007/s11082-020-02295-8

\begin{abstract}
We report on a numerical study of Cavity Resonator Integrated Grating Filters (a grating coupler flanked with two distributed Bragg reflectors) under oblique incidence. At resonance, the diffracted energy is divided equally in four outcoupling directions, versus two directions only for the CRIGF under normal incidence and for the infinite grating coupler. We provide a simple model to demonstrate this fact. Then, we propose a configuration for which all the diffracted energy is reflected backward with respect to the direction of the incident beam, thus realizing a reflection filter free from specular reflectivity.
\end{abstract}

\section{Introduction}

Multilayer interference filters are the most common way to achieve spectral filtering. But, to be efficient, they require a large number of optical layers, which make them difficult and expensive to build, especially for infrared applications. An alternative is nanostructured components, like Guided-Mode Resonant Filters (GMRF), which allow an ultranarrow spectral filtering with a few number of optical layers [1]. GMRF rely on the excitation of a guided mode by the incident light thanks to a subwavelength grating coupler (GC). The resonance wavelength of the structure is directly linked to the period of the GC. The main issue of this kind of structure is their lack of angular acceptance [2, 3]. Indeed, to have an efficient excitation of the guided mode, the incident beam must be perfectly collimated and the interaction area with the device must be large, otherwise the resonance peak is broaden and falls down.

A few years ago, a new type of structure has been proposed to generate resonance peaks spectrally as narrow as obtained with GMRF, but with a much greater angular acceptance: the Cavity Resonator Integrated Grating Filter 
(CRIGF) $[4,5,6]$. A CRIGF consists of a grating coupler, like in the GMRF case, integrated on a waveguide resonator formed by two Distributed Bragg Reflectors (DBR), as shown in figure 1. Under normal incidence, there is a peak in the reflectivity spectrum at the resonance wavelength. This peak can go to a maximum of $90 \%$ with a spectral width less than $1 \mathrm{~nm}$. CRIGF can be used as an end mirror in a laser cavity to stabilize the emission wavelength of a laser diode $[7,8]$.

The behavior of CRIGF under normal incidence is now quite well understood. Using CRIGF under oblique incidence could be of interest for some applications. During the first semester of 2019, two conference papers reported on CRIGF for oblique incidence, the first one from our team, and the second one from S. Ura's team $[9,10]$. In the present paper, our goal is to bring a further theoretical analysis of the behavior of CRIGF under oblique incidence. In the first part, using a rigorous numerical method, we show that there are four outcoupling directions of the guided mode when the CRIGF is illuminated under oblique incidence. We then explain theoretically this behavior, using a phenomenological approach involving the pole of the excited mode. Last, we study a configuration to control the emission directions of this CRIGF. We show an example where the incident light is quasi-totally backward reflected with respect to the direction of the incident beam.

\section{CRIGF under oblique incidence}

Figure 1 shows the CRIGF structure studied in this paper. The waveguide layer is made of $\mathrm{Si}_{3} \mathrm{~N}_{4}$ (refractive index 1.97) on a substrate of $\mathrm{Su}-8$ polymer (refractive index 1.6). The different gratings are etched in a $\mathrm{SiO}_{2}$ layer (refractive index 1.46).

The GC is designed to couple the incident wave into the $\mathrm{TE}_{0}$ mode of the structure through the -1 diffracted order. The period $\Lambda_{G C}$ of the GC is calculated thanks to the coupling equation under oblique incidence for a grating periodic along one direction only:

$$
\pm n_{e f f}=\sin \theta-m \frac{\lambda}{\Lambda_{G C}},
$$

where $m$ is the diffraction order used for the coupling, $n_{\text {eff }}$ is the effective index of the mode $\mathrm{TE}_{0}, \theta$ the incidence angle and $\lambda$ the wavelength. The sign \pm means that the mode can propagate forward $(+)$ or backward $(-)$ along $x$. The incident angle is chosen to be $30^{\circ}$. To obtain a resonance peak around $850 \mathrm{~nm}$ from the excitation of the mode propagating backward along $x\left(-n_{e f f}\right)$, with the first diffraction order, the period of the grating coupler (GC) is calculated to be $\Lambda_{G C}=390 \mathrm{~nm}$. The DBR period $\Lambda_{D B R}$ is chosen to couple the mode propagating in the positive direction (with a normalized propagation constant $+n_{\text {eff }}$ ) with the mode propagating in the negative direction (with a normalized propagation constant $-n_{\text {eff }}$ ), thus:

$$
\Lambda_{D B R}=\frac{\lambda}{2 n_{e f f}},
$$


from which we deduce $\Lambda_{D B R}=260 \mathrm{~nm}$.

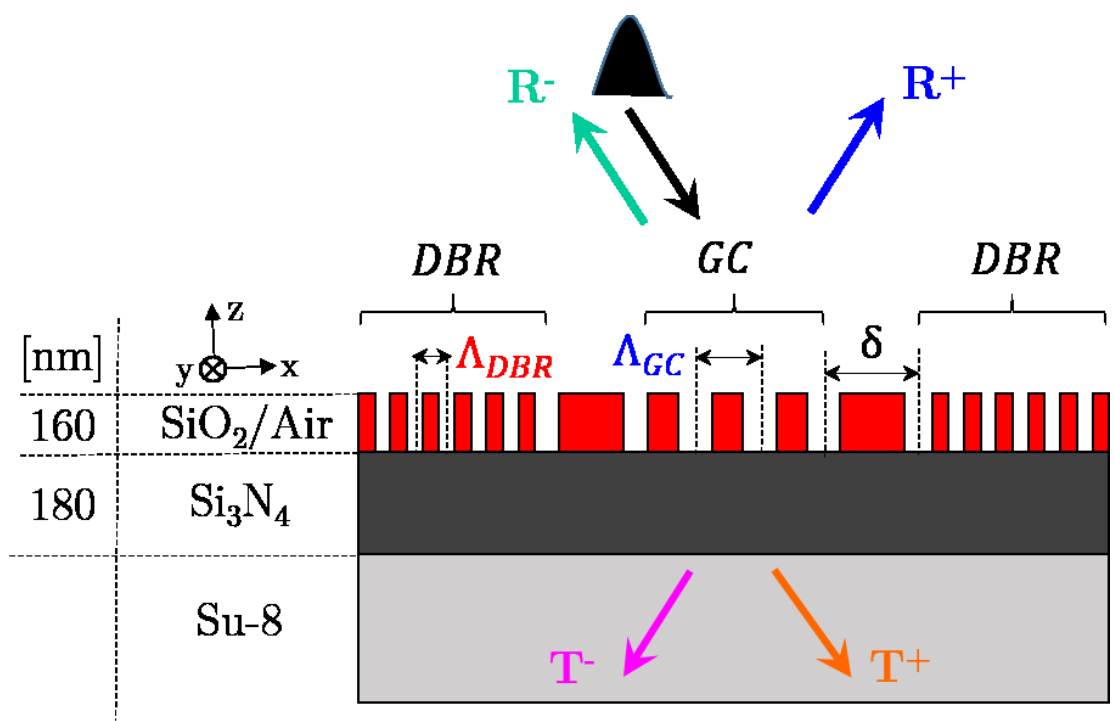

Figure 1: Scheme of the CRIGF structure under oblique incidence.

The GC is made of 21 periods with a filling factor of 0.45 , and the DBR are made of 300 periods with a filling factor of 0.5 . The phase section $\delta$ is used to change the length of the cavity between the two DBR, to adjust the spectral position of the resonance. The structure is simulated with the Fourier Modal Method (FMM) with 1801 Fourier coefficients (from -900 to 900) [11]. The incident beam is $4 \mu \mathrm{m}$ waist, and covers completely the GC (the length of the GC is $8.19 \mu \mathrm{m})$. When the structure is illuminated with an oblique incident Gaussian beam (illumination angle of $30^{\circ}$ ), the light is outcoupled in four different directions: $R^{+}, R^{-}, T^{+}, T^{-} \cdot R^{+}$and $T^{+}$are the specular reflection and transmission directions. They can be seen as the sum of a non-resonant term (which corresponds to the energy reflected and transmitted by the multilayer stack and that is not coupled into the guided mode) and a resonant term, which is the signal outcoupled from the waveguide layer at the resonance wavelength. The directions $R^{-}$and $T^{-}$correspond to the symmetric of $R^{+}$and $T^{+}$with respect to the $\mathrm{z}$ axis. They are free from non-resonant terms (not in the specular directions of reflection and transmission by the multilayer stack).

Figure 2 shows the reflectivity and transmittivity spectra of this CRIGF in each outcoupling direction calculated rigorously with the FMM. In dotted line, the total reflectivity (black) and total transmittivity (red) of the structure are plotted. One can see that the total reflectivity at the resonance is less than $50 \%$, which is different from the normal incidence case (around $90 \%$ of reflectivity at the resonance).

In solid line, the signals in the 4 outcoupling directions are plotted. At resonance, the reflectivity and transmittivity is almost equal to $25 \%$ in each direc- 


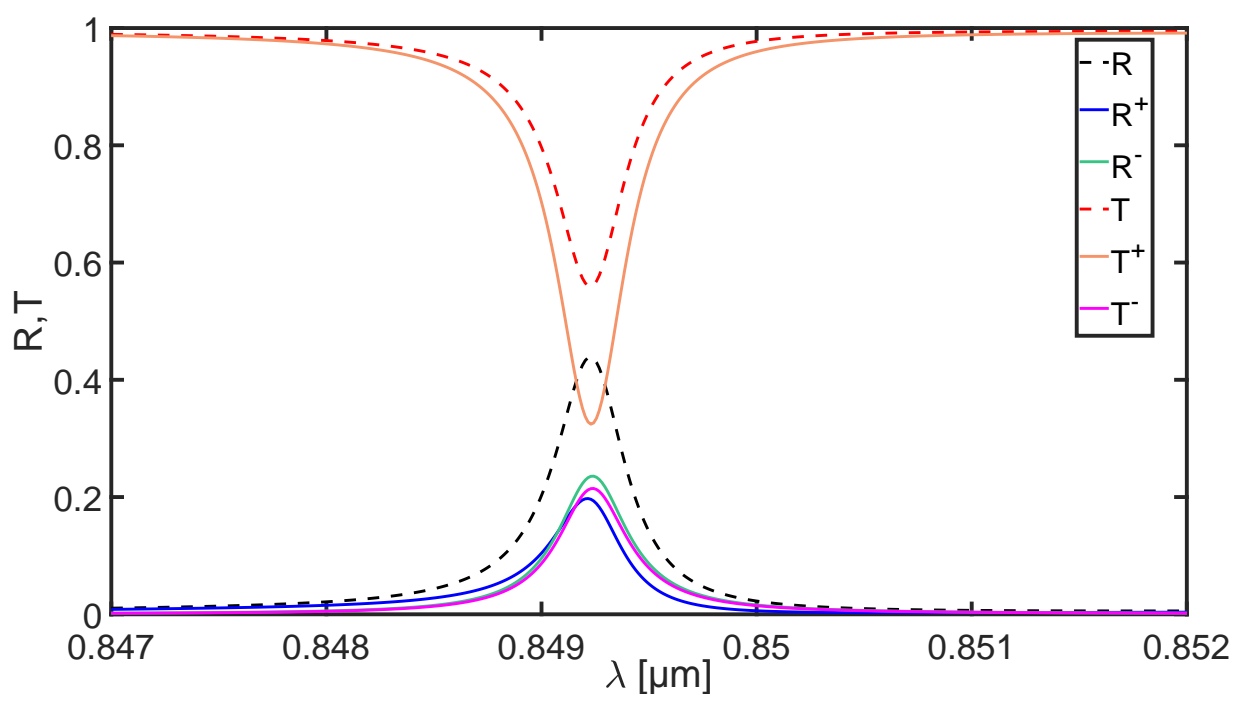

Figure 2: Reflectivity and transmitivity spectra of the CRIGF in each outcoupling direction.

tion, the device behaves as a beam splitter.

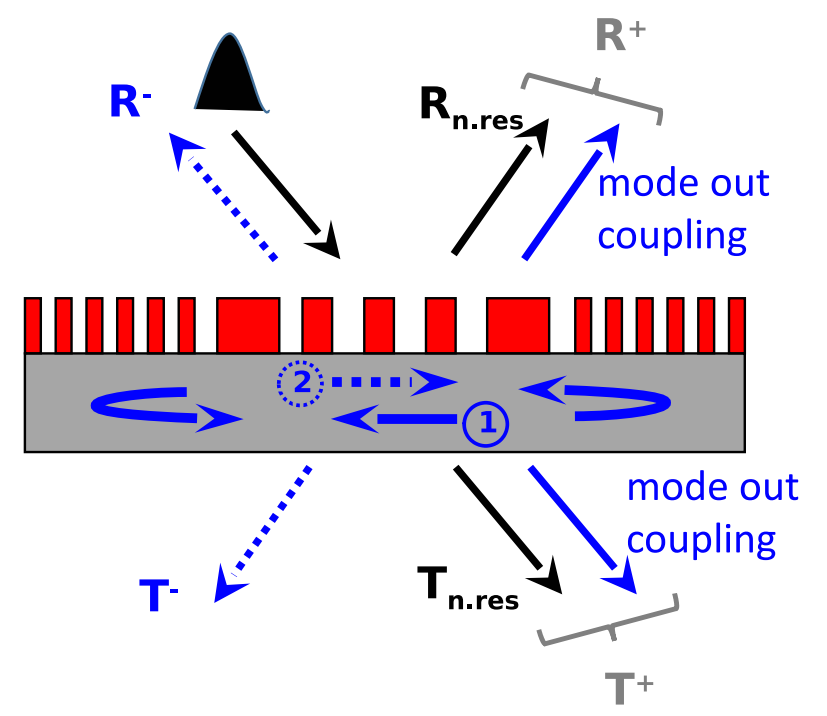

Figure 3: Scheme of the different contributions in each outcoupling direction. 
The outcoupling in the four directions can be understood by considering the different modes propagating inside the CRIGF (see figure 3). Indeed, when an incident beam is coming on the top of the structure (black arrow with the Gaussian symbol), a part of its energy is reflected and transmitted by the multilayer stack (non-resonant reflection $R_{n . r e s}$ and non-resonant transmission $T_{n . r e s}$ in black arrows) and the rest is coupled into one of the guided mode of the structure (solid blue arrow 1 in the gray layer) thanks to the -1 diffraction order of the grating coupler. The mode then propagates inside the waveguide layer until it reaches the left-hand side DBR. When going through the DBR, the mode is reflected in the opposite direction (dashed blue arrow 2 in the gray layer) and will be reflected by the right-hand side DBR. This configuration acts as a planar Fabry-Perot cavity for the guided mode, and the structure behaves as if there are two modes propagating in two opposites directions (solid and dashed arrows in the waveguide layer in gray), each of them carrying $50 \%$ of the initial energy. Following eq. 1, the mode represented with the arrow 1 (with normalized propagative constant $-n_{e f f}$ ) is outcoupled in the superstrate in the specular direction $R^{+}$, while the mode represented with the arrow 2 (with normalized propagative constant $\left.+n_{\text {eff }}\right)$ is outcoupled in the symmetric direction with respect to $z$, the direction $R^{-}$. And each of these two modes is also outcoupled in transmission ( $T^{+}$for mode 1 and $T^{-}$for mode 2 ).

In the next section, we demonstrate, using a phenomenological theory involving the pole associated with the resonance, that the energy of the mode is split equivalently in these four directions.

\section{Phenomenological theory for the CRIGF un- der oblique incidence}

In the case of a GMRF, the transmission of the structure represents a Fanotype anomaly [12] that can be written as the sum of a non-resonant transmission (transmission by the multilayer stack) and a Lorentzian resonant term $[13,14$, $15]$ :

$$
T_{0}=T_{n . r e s}+\frac{c_{\text {out }}^{t}}{\lambda-\lambda_{0}^{p}} g_{0}^{-},
$$

where $T_{n . r e s}$ corresponds to the transmission coefficient out of resonance, $c_{\text {out }}^{t}$ is the outcoupling coefficient in transmission, $g_{0}^{-}$is the amplitude of the mode excited, $\lambda_{0}^{p}$ is the eigenwavelength of the mode, solution of:

$$
\operatorname{det}[\mathbf{S}(\lambda)]^{-1}=0,
$$

with $\mathbf{S}$ the diffraction matrix of the GMRF.

A similar expression can be written for the reflectivity:

$$
R_{0}=R_{n . r e s}+\frac{c_{o u t}^{r}}{\lambda-\lambda_{0}^{p}} g_{0}^{-},
$$


where $R_{n . r e s}$ corresponds to the reflection coefficient out of the resonance and $c_{\text {out }}^{r}$ is the outcoupling coefficient in reflection.

For the following, we consider that

1. the non-resonant reflection is vanishing (multilayer designed to be an antireflection coating), and thus the non-resonant transmission modulus is unity,

2. the structure is symmetrical with respect to the $z$ axis.

In the case were the non-resonant reflection is vanishing, eq. 5 writes

$$
R_{0}=\frac{c_{o u t}^{r}}{\lambda-\lambda_{0}^{p}} g_{0}^{-} .
$$

From eq. 6, we can expect that the maximum of reflectivity is obtained for a wavelength close to the real part of the pole, $\lambda \simeq \operatorname{Re}\left(\lambda_{0}^{p}\right)$. Moreover, for a structure symmetrical with respect to the $z$ axis, it can be proved that the reflectivity maximum is $100 \%$ (provided that the materials are lossless [16]. Thus,

$$
R_{0}\left(\lambda=\operatorname{Re}\left(\lambda_{0}^{p}\right)\right)=i \frac{c_{\text {out }}^{r}}{\operatorname{Im}\left(\lambda_{0}^{p}\right)} g_{0}^{-}=\exp \left(i \psi^{r}\right),
$$

where $\psi^{r}$ is a real number. Thanks to the energy conservation, the transmittivity for $\lambda=\operatorname{Re}\left(\lambda_{0}^{p}\right)$ is null, which entails, from eq. 3, that

$$
i \frac{c_{\text {out }}^{t}}{\operatorname{Im}\left(\lambda_{0}^{p}\right)} g_{0}^{-}=-T_{n . r e s},
$$

where $T_{n . r e s}$ modulus is unity under the first hypothesis mentioned above.

Here the subscript 0 stands for the values for a GMRF. Now we consider a CRIGF. The transmittivity (resp. reflectivity) in the specular direction is, as in the case of the GMRF, the sum of the non-resonant transmittivity (resp. reflectivity) and a resonant term coming from the outcoupling of the mode. As the incident beam illuminates only the area covered by the GC, we can assume that the non-resonant part does not change significantly when shifting to the CRIGF geometry (thus $R_{n . r e s}$ is negligible while $T_{n . r e s}$ has a modulus equal to one). We also assume that the length of the GC is sufficient to efficiently couple in the mode, hence the coupling conditions are the same for the GMRF and the CRIGF, which means that neither the outcoupling coefficients nor the imaginary part of the pole $\lambda^{p}$ of the CRIGF are significantly different from those of the GMRF. Thus, following eq. 6 , we can write, for $\lambda=\operatorname{Re}\left(\lambda^{p}\right)$

$$
R^{+}\left(\lambda=\operatorname{Re}\left(\lambda^{p}\right)\right)=i \frac{c_{\text {out }}^{r}}{\operatorname{Im}\left(\lambda_{0}^{p}\right)} g^{-},
$$

and

$$
T^{+}\left(\lambda=\operatorname{Re}\left(\lambda^{p}\right)\right)=T_{n . r e s}+i \frac{c_{\text {out }}^{t}}{\operatorname{Im}\left(\lambda_{0}^{p}\right)} g^{-},
$$


where $g^{-}$is the amplitude of the mode propagating backward along $x$ inside the CRIGF.

In the negative direction, the reflection and transmission are simply due to the outcoupling of the mode propagating in the positive direction, and due to the symmetry, the outcoupling coefficients are the same in positive and negative direction. Hence, the $R^{-}$and $T^{-}$coefficients have the same form as that given by eqs. 9 and 10, except that there is no non-resonant terms and that $g^{-}$is replaced with $g^{+}$, the amplitude of the mode propagating forward along $x$ :

$$
R^{-}\left(\lambda=\operatorname{Re}\left(\lambda^{p}\right)\right)=i \frac{c_{\text {out }}^{r}}{\operatorname{Im}\left(\lambda_{0}^{p}\right)} g^{+},
$$

and

$$
T^{-}\left(\lambda=\operatorname{Re}\left(\lambda^{p}\right)\right)=i \frac{c_{\text {out }}^{t}}{\operatorname{Im}\left(\lambda_{0}^{p}\right)} g^{+} .
$$

Now, for a system symmetrical with respect to the origin, the two modes propagating backward and forward along $x$ in the CRIGF have the same amplitude, and each of them carries only half of the energy of the mode of GMRF considered above:

$$
g^{+}=g^{-}=\frac{1}{2} g_{0}^{-} .
$$

From eqs. 7, 9, 11 and 13, we obtain

$$
R^{+}\left(\lambda=\operatorname{Re}\left(\lambda^{p}\right)\right)=R^{-}\left(\lambda=\operatorname{Re}\left(\lambda^{p}\right)\right)=\frac{1}{2} \exp \left(i \phi^{r}\right),
$$

and from eqs. 8, 10, 12 and 13, we obtain

$$
T^{+}\left(\lambda=\operatorname{Re}\left(\lambda^{p}\right)\right)=-T^{+}\left(\lambda=\operatorname{Re}\left(\lambda^{p}\right)\right)=\frac{1}{2} T_{n . r e s},
$$

and their modulus is equal to 0.5 . In term of energy, it means that $25 \%$ of the energy goes in each of theses directions, as shown by the previous numerical results.

In the next part, we show how to obtain almost $100 \%$ of the energy in one of the four directions.

\section{Control of the emission direction of the CRIGF}

The control of four channels of emission is quite difficult. To return to a simpler case, where there are only two emission directions, a gold substrate is added at the bottom of the structure to remove the transmitted beams, as shown in figure 4. The signal $R_{T}^{+}$is the sum of the signal $R^{+}$and the signal $T^{+}$, which is reflected on the gold substrate. $R_{T}^{-}$corresponds to the sum of the signal $R^{-}$ and the signal $T^{-}$(also reflected on the gold substrate).

With this structure, our goal is to obtain a total reflection in the $R_{T}^{-}$direction (no signal in $R_{T}^{+}$). The control of the energy distribution between $R_{T}^{+}$and $R_{T}^{-}$ 


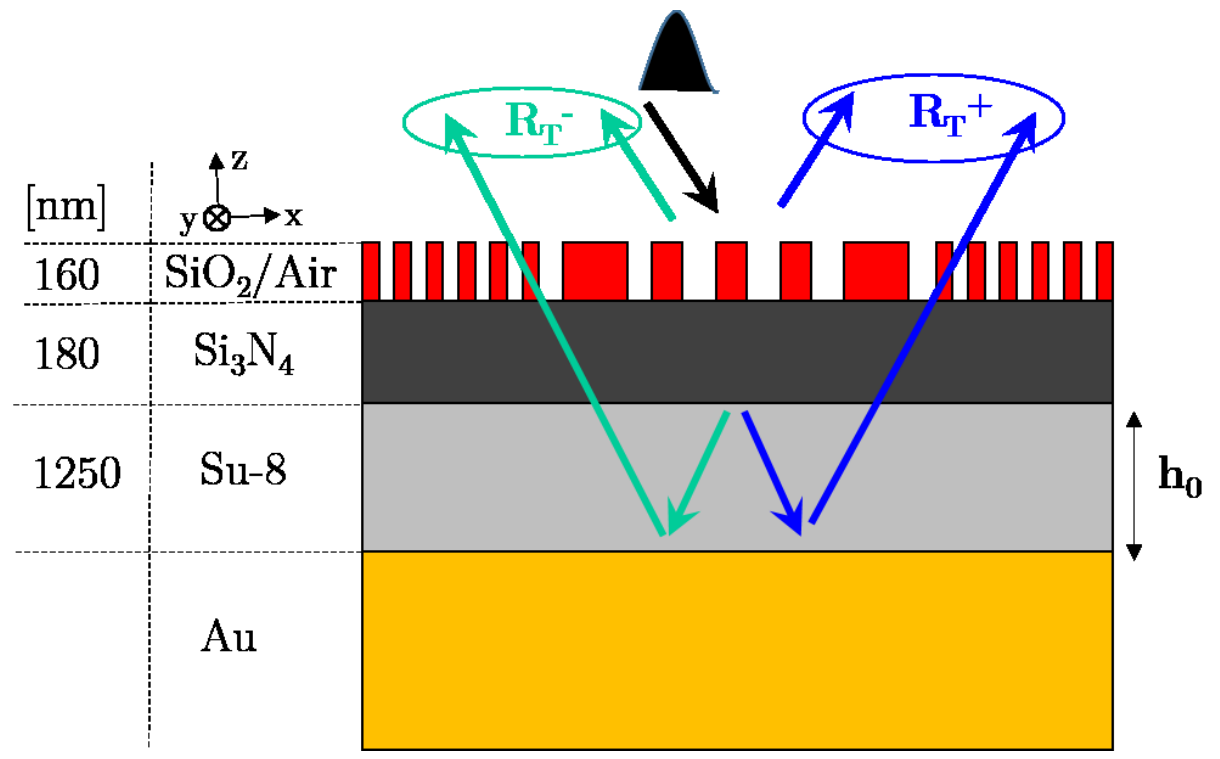

Figure 4: Scheme of the CRIGF with a gold substrate to control the emission direction

can be done by tuning the thickness of the buffer layer of $\mathrm{Su}-8$. By varying this thickness, the phase of the signal $T^{+}$(respectively $T^{-}$), which is reflected by the gold substrate, can be changed to interfere constructively or destructively with the signal $R^{+}$(respectively $R^{-}$).

In figure 5 , the reflected signals $R_{T}^{+}$and $R_{T}^{-}$are plotted versus the thickness of the Su- 8 buffer layer. The first thing to be noticed is that the $R_{T}^{+}$signal (blue) is in phase opposition with the $R_{T}^{-}$signal (green). This can be explained by the composition of these two signals. Indeed, the $R_{T}^{+}$signal contains the nonresonant specular reflection, in addition to the outcoupling of the mode. The $R_{T}^{-}$ signal contains only the resonant part that comes from the mode outcoupling.

The thickness of the Su- 8 buffer layer is chosen to be approximately $1250 \mathrm{~nm}$, which corresponds to a maximum of the $R_{T}^{-}$signal and a minimum of the $R_{T}^{+}$ signal. The reflectivity spectrum of this structure is plotted on figure 6 .

Far from the resonance, the reflectivity in $R_{T}^{+}$is maximum, and corresponds to the non-resonant reflection due to the multilayer stack and the gold mirror. The signal in $R_{T}^{-}$is very weak, because the modes are not excited inside the structure, but not null due to the scattering of the incident beam at the GC edges.

At the resonance wavelength (around $849 \mathrm{~nm}$ ), the signal coming from the outcoupling of the mode interferes destructively with the non-resonant part in the direction $R_{T}^{+}$, while the signal becomes maximum in the $R_{T}^{-}$direction: the incident light is quasi-totally backward reflected by the CRIGF.

We think that this configuration can be interesting when compared to the 


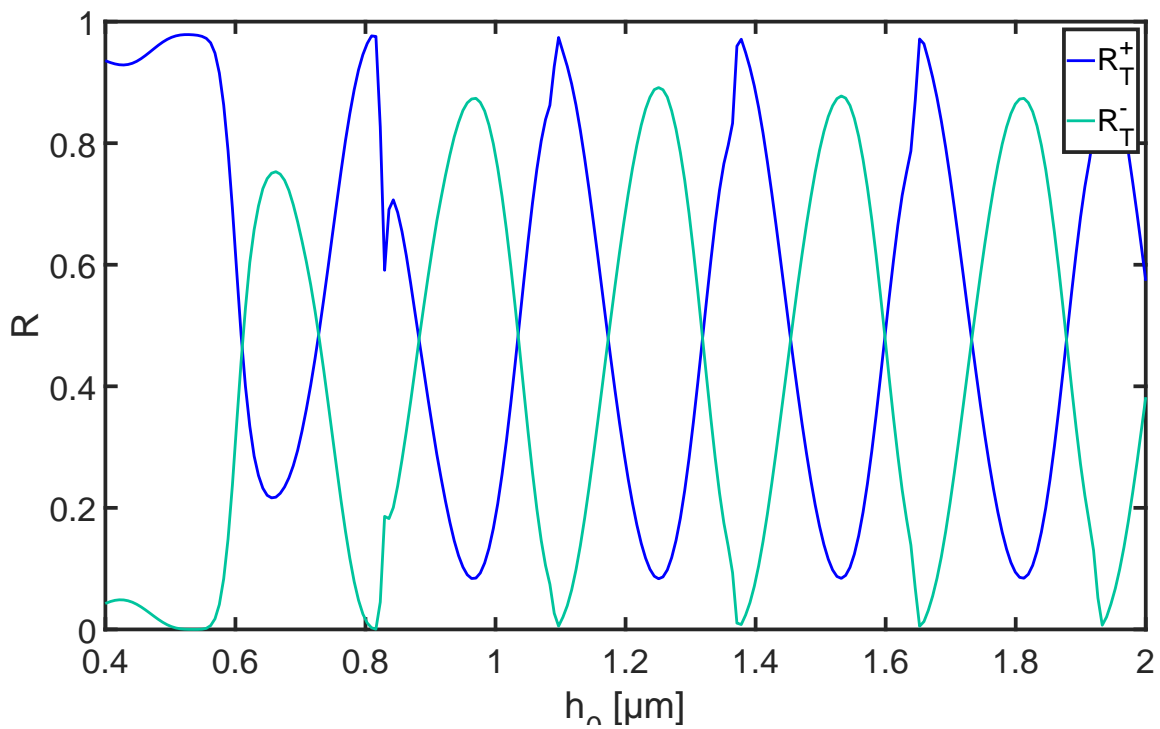

Figure 5: Evolution of the reflected signals $R_{T}^{+}$and $R_{T}^{-}$versus the thickness $\mathrm{h}_{0}$ of the Su-8 buffer layer.

CRIGF used in normal incidence, because in the $R_{T}^{-}$direction, there is no FabryPerot interferences due to the substrate thickness, which are parasitic signals for filtering applications (for instance, the oscillations on the fig. 2 of [17] are due to the interferences coming from the thick substrate).

\section{Conclusion}

In this paper, we studied the behavior of the CRIGF under oblique incidence. Numerically, we show that the modes in the structure are outcoupled in four different directions, and that the energy in each of these directions is approximately the same. We gave a demonstration of this result thanks to a phenomenological approach. In order to reduce the number of outcoupling paths, we added a metallic mirror at the bottom of the structure, and show that a particular choice of the distance between the mirror and the waveguide lead to an outcoupling of $80 \%$ of the energy in the backward direction with respect to the incident beam. The main advantage of this configuration is to produce at resonance a reflected signal free from interference due to the substrate.

F. Renaud acknowledges PhD grant from DGA/AID. 


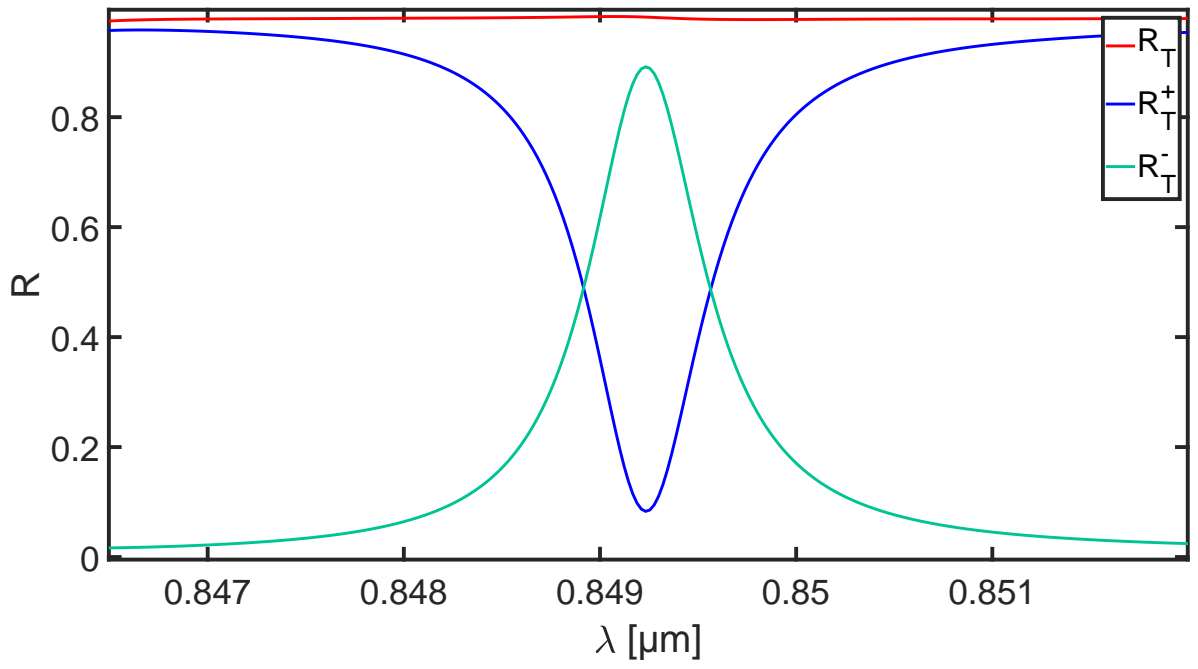

Figure 6: Reflectivity spectrum of the CRIGF in the quasi-total backward reflection configuration.

\section{References}

[1] S.S. Wang, R. Magnusson, Applied Optics 32(14), 2606 (1993). DOI 10.1364/AO.32.002606. URL https://www.osapublishing.org/ao/abstract.cfm?uri=ao-32-14-2606

[2] F. Lemarchand, A. Sentenac, E. Cambril, H. Giovannini, Journal of Optics A: Pure and Applied Optics 1(4), 545 (1999)

[3] A.L. Fehrembach, A. Talneau, O. Boyko, F. Lemarchand, A. Sentenac, Optics letters 32(15), 2269 (2007)

[4] S. Ura, J. Inoue, K. Kintaka, Y. Awatsuji, in 2011 13th International Conference on Transparent Optical Networks (IEEE, 2011), pp. 1-4

[5] K. Kintaka, T. Majima, J. Inoue, K. Hatanaka, J. Nishii, S. Ura, Optics express 20(2), 1444 (2012)

[6] N. Rassem, A.L. Fehrembach, E. Popov, JOSA A 32(3), 420 (2015)

[7] X. Buet, E. Daran, D. Belharet, F. Lozes-Dupuy, A. Monmayrant, O. Gauthier-Lafaye, Optics express 20(8), 9322 (2012) 
[8] X. Buet, A. Guelmani, A. Monmayrant, S. Calvez, F. Lozes-Dupuy, O. Gauthier-Lafaye, in 2013 15th International Conference on Transparent Optical Networks (ICTON) (IEEE, 2013), pp. 1-2

[9] T. Kusuura, K. Akamatsu, J. Inoue, K. Kintaka, S. Ura, (2019). URL https://confit.atlas.jp/guide/event/oeccpsc2019/subject/ThE1$2 /$ advanced

[10] F. Renaud, A.L. Fehrembach, E. Popov, A. Monmayrant, O. GauthierLafaye, (2019). URL http://www.owtnm2019.com/program

[11] N. Rassem, E. Popov, A.L. Fehrembach, Optical and Quantum Electronics $\mathbf{4 7}(9), 3171(2015)$

[12] U. Fano, J. Opt. Soc. Am. A 31(3), 213 (1941)

[13] A.L. Fehrembach, B. Gralak, A. Sentenac, Physical Review A 97(4), 043852 (2018)

[14] S. Fan, J.D. Joannopoulos, Phys. Rev. B 65, 235112 (2002)

[15] P. Paddon, J.F. Young, Opt. Lett. 23(19), 1529 (1998)

[16] E. Popov, L. Mashev, D. Maystre, Optica Acta: International Journal of Optics 33(5), 607 (1986)

[17] S. Augé, A. Monmayrant, S. Pelloquin, J.B. Doucet, O. Gauthier-Lafaye, Optics express 25(11), 12415 (2017) 